\title{
The eliminatory effects of cold argon plasma jet on aflatoxin B1 produced by different isolates of Aspergillus section nigri
}

\author{
S. Hassanpour ${ }^{1}$, M. Bayat ${ }^{1 *}$, A. Chaichi Nosrati ${ }^{2}$, M. Ghorannevis ${ }^{3}$, S. Hashemi ${ }^{4}$ \\ ${ }^{I}$ Department of Microbiology, Science and Research Branch, Islamic Azad University, Tehran, Iran \\ ${ }^{2}$ Department of Molecular \& Cell Biology, Lahijan Branch, Islamic Azad University, Lahijan, Iran \\ ${ }^{3}$ Plasma Physics Research Center, Science and Research Branch, Islamic Azad University, Tehran, Iran \\ ${ }^{4}$ Department of Medical Parasitology and Mycology, School of Public Health, Tehran University of Medical Science, \\ Tehran, Iran
}

Received December 16, 2017; Revised August 21, 2018

\begin{abstract}
Aflatoxin and ochratoxin are among the most resistant and severe toxins produced by Aspergillus spp. The aim of this study was an evaluation of eliminatory effects of cold argon plasma jet on aflatoxin B1. Sampling of wheat, corn, oatmeal, flour and rice products from northern cities of Iran was carried out. The fungal species were cultured on CHAPK medium and next to sabouraud dextrose broth + malt extract (SB + ME) and also SB + yeast extract (SB + YE) media to obtain aflatoxin B1. The ELISA test was conducted to measure the aflatoxin level. The mean initial concentration of the aflatoxin from charts analysis in the SB +ME medium was $16.106 \mu \mathrm{g} / \mathrm{kg}(10.4,8.32$ and $7.55 \mu \mathrm{g} /$ $\mathrm{kg}$ at 30, 60 and $360 \mathrm{sec}$, respectively), and in the $\mathrm{SB}+\mathrm{YE}$ medium from $23.699 \mu \mathrm{g} / \mathrm{kg}(12.82,9.93$ and $9.54 \mu \mathrm{g} / \mathrm{kg}$, respectively), at the same time. Furthermore, the mean concentrations of aflatoxin B1 by tables analysis in the SB+ ME medium changed from $20.02 \mu \mathrm{g} / \mathrm{kg}$ to $6.87,10.46$ and $7.55 \mu \mathrm{g} / \mathrm{kg}$, at 30,60 and $360 \mathrm{sec}$, respectively, and in the medium SB +YE from $28.85 \mu \mathrm{g} / \mathrm{kg}$ reached $6.56 .6,8.43$ and $7.02 \mu \mathrm{g} / \mathrm{kg}$, respectively, at the same time. In this study, the change or decrease in the concentration of aflatoxin B1, from Lin/Log and Log/Lin analyses of charts was significant $(\mathrm{p}<0.05)$ at $60 \mathrm{~s}$ and $360 \mathrm{~s}$ of application of the cold argon plasma jet in both media. The cold plasma as a new technology in various fields of food and agriculture can provide effective and suitable solutions in order to promote the goals of food industry, especially in the areas of mycotoxin elimination.
\end{abstract}

Keywords: Aspergillus nigri, Aflatoxins, Cold plasma jet, ELISA

\section{INTRODUCTION}

Aspergillus species are classified among the most important group of pathogenic and destructive fungi with over 900 species present, and 50 of which produce toxic metabolites. The economic importance of this genus is also because of the production of acids or enzymes by a number of species [1]. Aspergillosis, a disease caused by these species, is a major cause of degradation of agricultural products before and after cultivation via the production of various mycotoxins, and this is the most crucial harmful effect. The species produce highly potent toxins with long-lasting and severe effects $[2,3]$. The most important toxins of this species which function in human and animal food industries include aflatoxin, ochratoxin A, citrinin, cyclopiazonic acid, patulin, sterigmatocystin and some other thermogenic toxins $[4,5]$.

Mycotoxins, along with other fungal metabolites, such as antibiotics and alkaloids, are compounds produced by the fungal cells in the late stages of growth of the fungus. These secondary metabolites apparently act as a defense mechanism and are produced in human and animal food when suitable conditions exist. Obtaining of polyacetyl-derived mycotoxins is the most important pathway for mycotoxin biosynthesis due to the involvement of acetyl coenzyme A, which produces mycotoxins such as aflatoxin, ochratoxin, patulin, citrinin, and pentanedioic acid $[6,7]$.

The most important mycotoxins produced by Aspergillus species include aflatoxin and ochratoxin. Aflatoxins are a large group of mycotoxins produced by certain species of Aspergillus. This group of fungal toxins is considered the head of all mycotoxins [8]. These toxins are common pollutants of cereals and oilseeds, starch, dried fruit, and coffee. The metabolites produced by aflatoxins include aflatoxin M1 and M2. Aflatoxin M1 and M2 are mono-hydroxyl derivatives of aflatoxins $\mathrm{B} 1$ and B2, respectively, stored in human milk and lactating animals and formulated and secreted through diets contaminated of aflatoxins B1 and B2 [9].

The aflatoxin $\mathrm{B} 1$ has a molecular weight of 312 Dalton and its structural formula is $\mathrm{C}_{17} \mathrm{H}_{12} \mathrm{O}_{6}$. This compound is destroyed at $268-269{ }^{\circ} \mathrm{C}$ without melting. Its maximum optical absorption is at 265 , 262, and $223 \mathrm{~nm}$. Aflatoxins, in spite of the high resistance to human and animal nutrition, are inactive at $\mathrm{pH}$ values above 10 and below 3 . Likewise, oxidizing compounds and ultraviolet

* To whom all correspondence should be sent:

E-mail: Dr_mansour_bayat@yahoo.com

(C) 2019 Bulgarian Academy of Sciences, Union of Chemists in Bulgaria 
S. Hassanpour et al.: The eliminatory effects of cold argon plasma jet on aflatoxin $B 1$ produced by different isolates ...

radiation in the presence of oxygen cause the inactivation of some types of aflatoxins. According to the food and drug organization (FAO) annual report, $20 \%$ of the world's food products are contaminated by fungal toxins, mostly including aflatoxins $[10,11]$.

The plasma is, in fact, the fourth state of matter and is a set of particles produced by an energy source such as an electrical power supply in the environment. The cold or non-thermal plasma is a kind of plasma that can be produced simply and quickly using gas evacuation. The use of plasma systems is a new method of sterilization. The ability of sterilization at relatively low temperatures and in short periods leads to non-toxicity of the plasma. In this way, all microorganisms are killed without causing pathogenesis. In plasma sterilization, in addition to killing bacteria, dead bacteria are removed from the surface of the material due to a process similar to that of plasma engraving [12-14].

Until now, efforts have been performed to use different types of plasma to eliminate existing microorganisms in the food. However, no research has been conducted in Iran on the use of atmospheric plasma jet to mycotoxins elimination. The aim of this study was to investigate the effects of cold argon plasma jet on aflatoxin B1 produced by different isolates of Aspergillus nigri.

\section{METHODOLOGY}

\section{Sampling}

Sampling of wheat, corn, oatmeal, flour and rice products from the northern cities of Iran was carried out. Samples were transferred to the laboratory immediately after collection in order to determine the amount of aflatoxin B1 and to monitor the effect of plasma jet on mycotoxin at $4^{\circ} \mathrm{C}$.

\section{Culture conditions}

In this study, a photochemical culture medium was used for enrichment, isolation, and production of mycotoxin of Aspergillus from Sabouraud dextrose agar broth environments, malt extract and yeast extract (Merck, Germany).

Culturing the specimens in a CHAPK agar medium. For isolation of aflatoxin $\mathrm{B} 1$ bearing fungi, the samples were cultured in a CHAPK agar medium and incubated for 2 weeks at $25{ }^{\circ} \mathrm{C}$. In order to identify fungi, their morphological characteristics were determined, such as colony color, landscape view of the colony for the presence of folding and breaker, radial lines or concentric circles, smooth or folded colony levels, and also the colony level with respect to the identification keys of Barnett and Hunter, Click and Dugan.
Cultivation in a liquid medium. In order to increase mycotoxin production, the sabouraud dextrose broth + malt extract $(\mathrm{SB}+\mathrm{ME})$ and also sabouraud dextrose broth + yeast extract $(\mathrm{SB}+\mathrm{YE})$ media were applied. Isolated species were transferred to the liquid medium and incubated at $25^{\circ} \mathrm{C}$ for 2 weeks in a shaker incubator. To prevent drying of liquid media, phosphate salt buffer was added to the culture media.

Preparation of slide culture from selected fungal species. The slide culture was prepared for each species previously grown in CHAPK media. Using a sterile scalp, a portion of the medium was cut into square centimeters and placed on a slide. Then, it was put on a U-shaped tube inside a glass plate; this U-shaped tube was used to prevent the contact of the plate with the slide on it. Then the isolation of species was inoculated into 4 dots of the medium and the sterilized small slide on the tray. The agar was incubated for 14 days at $25{ }^{\circ} \mathrm{C}$. After growth, the fungal species were investigated using lactophenol cotton blue on the slide under the optical microscope.

\section{Extraction of mycotoxins from culture media}

After incubation for two weeks, extraction and purification of mycotoxins were performed from pure strains in a non-cellular medium. For this purpose, the tubes containing the liquid medium were subjected to vortex with an angle of 35 degrees for at least $15 \mathrm{~min}$ and then kept at $-70^{\circ} \mathrm{C}$. After defrosting at the laboratory temperature, 2.5 $\mathrm{ml}$ of extraction solvent was added and the sample was vortexed twice for $15 \mathrm{~min}$. At the final stage, the mycotoxins were separated from the supernatant using a funnel and filter paper, transferred into a sterile micro-tube and stored at $4^{\circ} \mathrm{C}$ for further study.

Extraction of mycotoxins from the CHAPK medium. The CHAPK agar was cut by a sterile scalpel to $3 \times 1 \mathrm{~cm}$ sized portions, inoculated into a sterile falcon tube $(10 \mathrm{cc})$ containing $5 \mathrm{cc}$ of phosphate salt solution and placed in a freezer at 70 ${ }^{\circ} \mathrm{C}$ after being vortexed for a minimum of $15 \mathrm{~min}$. The next step, the same as the extraction of mycotoxin from the broth medium, was performed using an extraction solvent and Whatman filter paper.

\section{Analysis of aflatoxin B1 by ELISA test}

The ELISA test was implemented with the Biotech-ELX 800 device. An enzyme kit and aflatoxin B1 standards were provided from the RBiopharm Corporation, Germany. Aflatoxin B1 kits (R1211) contain competitive immunoassay enzyme to determine the amounts of these toxins in foods. 


\section{S. Hassanpour et al.: The eliminatory effects of cold argon plasma jet on aflatoxin B1 produced by different isolates ...}

The extraction protocol was used according to the instruction.

Accordingly, $50 \mu \mathrm{l}$ of the standard aflatoxin solutions $(0,1,5,10,20$ and $50 \mathrm{ppb})$ and $50 \mu \mathrm{l}$ of the specimen were added separately to microtiter wells in duplicate. Then $50 \mu \mathrm{l}$ of the conjugated enzyme was added to each well and incubated for $10 \mathrm{~min}$ at $20-25^{\circ} \mathrm{C}$. In the next step, $50 \mu \mathrm{L}$ of antiaflatoxin antibody solution was added to each well and incubated at 25 to $20{ }^{\circ} \mathrm{C}$. Wells were washed three times with deionized water. In the next step, $100 \mu \mathrm{l}$ of the substrate / chromogenic solution was added to each well and incubated in dark space at 20-25 ${ }^{\circ} \mathrm{C}$. In the final step, $100 \mu \mathrm{l}$ of the stopping solution was added and after $10 \mathrm{~min}$, absorption was measured with ELISA reader at OD $=450 \mathrm{~nm}$. The standard calibration curve was drawn based on the percentage of absorption of standard samples and the amount of aflatoxin in the samples. Subsequently, aflatoxin content of each sample was obtained on the basis of the absorbance of each sample and its correlation with the calibration curve in a $\mu \mathrm{g} / \mathrm{kg}$ scale.

\section{Elimination of mycotoxins with the cold atmospheric plasma jet}

Argon gas was used to create the plasma and to eliminate mycotoxins. In this device, the chamber in which the plasma is formed is helix-shaped to ensure better contact of the plasma jet with the level of mycotoxin. The length of the helix pipe is $12 \mathrm{~cm}$ and its internal and external diameter is 2 and 3 $\mathrm{mm}$, respectively. Its core diameter is $3 \mathrm{~cm}$ and its distance is $1.5 \mathrm{~cm}$. The two ends of the helix are divided into three ways to allow the simultaneous entry of gas and mycotoxin and can be separated from each other at the exit site.

The output of the power supply was adjusted to $50 \mathrm{kV}, 100$ Watt and the electron frequency to 30 $\mathrm{kHz}$, and its values were measured with high voltage probes and oscilloscope. Then, at a gas flow rate of 6 liters per min and a high potential difference between the two electrodes, the effect of the cold atmospheric argon gas plasma jet were investigated at 30,60 and $360 \mathrm{sec}$.

\section{Statistical analysis}

The obtained data were analyzed by SPSS 18 software and one-way ANOVA for comparison of aflatoxin B1 levels in wheat, corn, barley, flour and rice samples. P-value $<0.05$ determined a significant result.

\section{RESULTS}

\section{Adoption of mycotoxin producing Aspergillus spp.}

In this study, five different food sources (wheat, corn, oats, flour, and rice) were sampled and 12 different Aspergillus species were identified among them. To compare the results and control the production of mycotoxins among isolates, the $A$. nigri standard strain was used.

ELISA assay. The ELISA assay was implemented before and after the application of the cold atmospheric plasma jet pressure on aflatoxin B1 mycotoxin. The concentration of aflatoxin B1 was higher than the limit determined in the national standard of Iran among more than $90 \%$ of wheat, corn, oatmeal, flour and rice samples. The permitted concentration of aflatoxin B1 according to the national standard of Iran, entitled "Human Feed - Livestock - Maximum Tolerability of Mycotoxins", with the number: 5925 is equal to 5 ppb.

\section{Aflatoxin B1 concentration measurement by the analysis of charts by Lin/Log and Log/Lin analysis}

The aflatoxin B1 concentration measurement was obtained by the analysis of Lin / Log charts in sabouraud dextrose broth + malt extract $(\mathrm{SB}+\mathrm{ME})$ and yeast extract $(\mathrm{SB}+\mathrm{YE})$.

The mean initial concentration of aflatoxin from charts analysis in the SB +ME medium was 16.106 $\mu \mathrm{g} / \mathrm{kg}$ and it was $10.4,8.32$ and $7.55 \mu \mathrm{g} / \mathrm{kg}$ at 30 , 60 and $360 \mathrm{sec}$, respectively. In the SB +YE medium, from $23.699 \mu \mathrm{g} / \mathrm{kg}$ it reached 12.82, 9.93 and $9.54 \mu \mathrm{g} / \mathrm{kg}$, respectively, at the same time.

Furthermore, the mean concentration of aflatoxin B1 by tables analysis of $\log /$ Lin charts in SB+ ME medium altered from $20.02 \mu \mathrm{g} / \mathrm{kg}$ to 6.87 , 10.46 and $7.55 \mu \mathrm{g} / \mathrm{kg}$, at 30,60 and $360 \mathrm{sec}$, respectively, and in the SB +YE medium from $28.85 \mu \mathrm{g} / \mathrm{kg}$ it reached 6.56.6, 8.43 and $7.02 \mu \mathrm{g} /$ $\mathrm{kg}$, respectively, at the same time.

As noted above, the change or decrease in the concentration of aflatoxin B1, from $\mathrm{Lin} / \mathrm{Log}$ and $\mathrm{Log} / \mathrm{Lin}$ analyses of charts was significant $(\mathrm{p}<0.05)$ at $60 \mathrm{~s}$ and $360 \mathrm{~s}$ of the application of the cold argon plasma jet in both media and likewise, from the analysis of tables results, it was significantly decreased at all the times at which the cold argon gas plasma jet was applied. The Log/Lin, Lin/Log results and their comparison are shown in diagrams 1,2 and 3. The concentrations of aflatoxin in dextrose broth + malt extract and dextrose broth + yeast extract are shown in Table 1.

According to the obtained data from the analysis of $\mathrm{Log} / \mathrm{Lin}$ and $\mathrm{Lin} / \mathrm{Log}$ aflatoxin B1, at 30 and 360 $\mathrm{s}$, the $\mathrm{Log} / \mathrm{Lin}$ analysis exhibits a stronger decrease compared to the Lin/Log analysis. However, at the $60 \mathrm{~s}$ treatment, the Lin/Log analysis demonstrated a stronger decrease than $\mathrm{Log} / \mathrm{Lin}$ analysis. According to the results analysis, the $\operatorname{Lin} / \log$ showed that 
S. Hassanpour et al.: The eliminatory effects of cold argon plasma jet on aflatoxin B1 produced by different isolates ... aflatoxin after $60 \mathrm{sec}$ in the ME+SB decreased by $56 / 65 \%$ and in YE+SB decreased by $41.85 \%$. Likewise, after $360 \mathrm{~s}$ in the ME+SB $46.87 \%$ and from $60 \mathrm{~s}-360 \mathrm{~s}$ it decreased by $9.25 \%$. Moreover, the reduction of toxin in the YE+SB after $360 \mathrm{~s}$ was $40.26 \%$ and after $60 \mathrm{~s}-360 \mathrm{~s}$ the reduction was $3.83 \%$

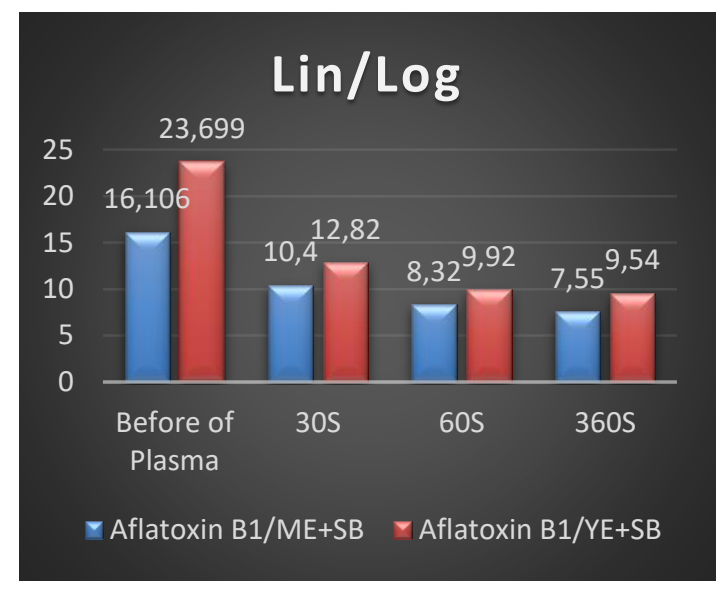

Diagram 1. Average concentration of aflatoxin B1 before and after exposure to plasma (Lin/Log results)

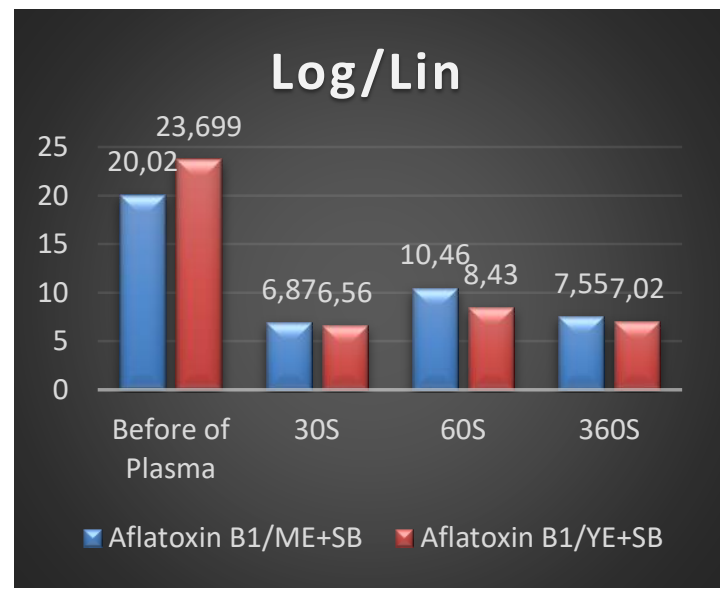

Diagram 2. Average concentration of aflatoxin B1 before and after exposure to plasma ( $\mathrm{Log} / \mathrm{Lin}$ results)

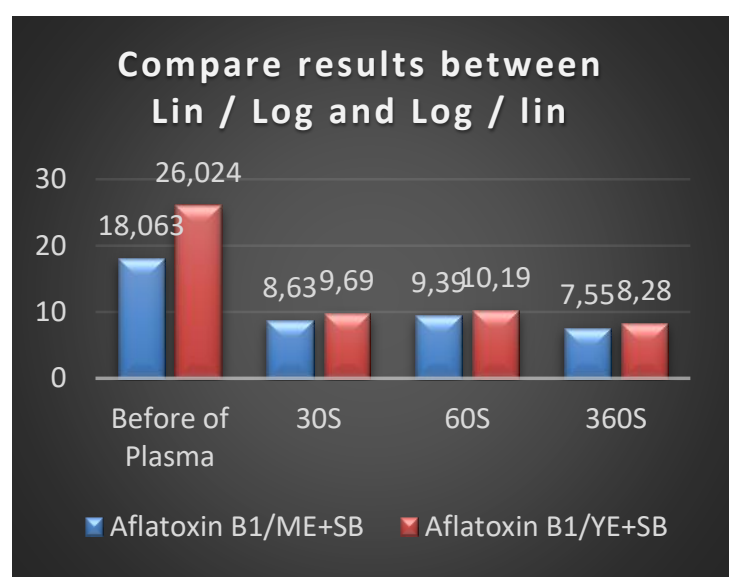

Diagram 3. Average concentration of aflatoxin B1 before and after exposure to plasma (Comparison of results between $\operatorname{Lin} / \log$ and $\log / \operatorname{Lin}$ )
Table 1. Concentrations of aflatoxin in dextrose broth + malt extract and dextrose broth + yeast extract

\begin{tabular}{|c|c|c|c|c|}
\hline \multirow{3}{*}{ SB+ME } & \multicolumn{4}{|c|}{ AFB1 } \\
\cline { 2 - 5 } & & $\mathrm{c} / \mathrm{m} 30$ & $\mathrm{c} / \mathrm{m} 60$ & $\mathrm{c} / \mathrm{m} \mathrm{360}$ \\
\cline { 2 - 5 } & Lin/Log & 7.87 & 8.78 & 8.78 \\
\cline { 2 - 5 } & $\mathrm{Log} / \mathrm{Lin}$ & 6.87 & 10.46 & 7.55 \\
\hline \multirow{3}{*}{$\mathrm{SB}+\mathrm{YE}$} & & $\mathrm{c} / \mathrm{y} 30$ & $\mathrm{c} / \mathrm{y} 60$ & $\mathrm{c} / \mathrm{y} 360$ \\
\cline { 2 - 5 } & $\mathrm{Lin} / \mathrm{Log}$ & 7.73 & 7.29 & 7.29 \\
\cline { 2 - 5 } & $\mathrm{Log} / \mathrm{Lin}$ & 6.56 & 8.43 & 7.02 \\
\hline
\end{tabular}

\section{DISCUSSION}

The inhibitory effect of the cold plasma was firstly demonstrated by Kung et al. (2009) against bacteria [15]. The cold atmospheric plasmas have the ability to inactivate microorganisms under controlled laboratory conditions. In 2001, Moissan et al. performed sterilization using jet plasma. They used a plasma jet machine at a frequency of $13 / 56$ $\mathrm{MHz}$ against a highly resistant spore-forming bacillus species [16].

In 2005, Larusi examined the sterilization using argon microwave plasma at atmospheric pressure at a frequency of $2.45 \mathrm{GHz}$ on Escherichia coli and Staphylococcus aureus bacterial species. The results of this study confirmed that the effects of this plasma at atmospheric pressure needed less sterilization time due to free radicals and ultraviolet radiation produced during plasma motility, for the high microwave plasma density, high free radicals and high ultraviolet light. The results showed that both bacteria were completely sterilized in less than one second, regardless of bacterial strains [17].

Bassaran observed that cold low-pressure plasma was developed using sulfur hexafluoride gases (SF6) and they tested it for its antifungal effect on Aspergillus parasiticus in nuts and treated them with both gas plasma and SF6 plasma for $20 \mathrm{~min}$. When the effect of plasma treatment on aflatoxins was tested, the gas plasma exhibited 50\% reduction in the total aflatoxins (AFB1, AFB2, AFG1, AFG2), but only $20 \%$ reduction was measured in the total aflatoxin by SF6 plasma treatment after 20 min. In these experiments, they concluded that the use of plasma is a more efficient approach to eliminate aflatoxin produced by fungi [18].

In our study, toxin decontamination (aflatoxin B1) by the cold plasma jet was investigated at atmospheric pressure of argon gas. In summary, it can be stated that many disinfection methods, including chemical, physical, dry or wet heat, are used to disinfect solid surfaces or liquids and biological surfaces such as skin with functional limitations. The use of non-aggressive plasma technology in cleansing microbes and toxin removal has advantages over existing methods, including high decontamination properties during 
S. Hassanpour et al.: The eliminatory effects of cold argon plasma jet on aflatoxin $B 1$ produced by different isolates ...

short flow, lack of remaining toxicity, and the costeffectiveness of argon gas. In the Pearson correlation ( Lin / Log) analysis of the aflatoxin B1 concentrations in $\mathrm{ME}+\mathrm{SB}$ and $\mathrm{YE}+\mathrm{SB}$ media after treatment with the plasma jet the aflatoxin $\mathrm{B} 1$ reduction in $\mathrm{ME}+\mathrm{SB}$ medium was stronger than that in the $\mathrm{YE}+\mathrm{SB}$ medium and there was a significant statistical correlation.

Furthermore, a significant correlation was observed between the levels of aflatoxin B1 toxicity and the increase in the plasma treatment time in both ME + SB and YE + SB media. The analysis of the results showed that aflatoxin $\mathrm{B} 1$ concentrations in $\mathrm{ME}+\mathrm{SB}$ and $\mathrm{YE}+\mathrm{SB}$ media decreased with increasing the cold plasma jet treatment time. As it was stated, the comparison of the reduction ratio and correlation of changes in the amount of aflatoxin $\mathrm{B} 1$ toxin in the two $\mathrm{ME}+\mathrm{SB}$ and $\mathrm{YE}+$ SB media after $60 \mathrm{sec}$, the plasma effect was statistically confirmed to be more efficient in the $\mathrm{ME}+\mathrm{SB}$ than in the YE + SB medium.

In similar studies in 2017, Devi et al. used the effect of cold plasma jet at atmospheric pressure with argon gas on the elimination of mycotoxins produced by Aspergillus species. In their study, they were able to remove more than $97 \%$ of aflatoxin B1 produced by Aspergillus parasiticus and more than 95 aflatoxins produced by Aspergillus flavus by plasma emission at 15 and 12 min, respectively [19].

Ouf et al. [20] employed the low-pressure cold plasma for toxin elimination in 2015 and demonstrated removal of both aflatoxin and ochratoxin produced by Aspergillus nigri at a rate of 3.5 and 7.5 liter per min, respectively [20].

\section{CONCLUSION}

According to the results obtained from this study, the non-thermal (cold) atmospheric plasma jet can exert fungicidal and mycotoxin eliminatory effects. Due to the presence of charged particles of electrons and ions, ultraviolet radiation, free radicals and reactive chemical species, the plasma can cause changes in the cell wall, morphology, or genetic characteristics of microorganisms and destroy them. As a result, the cold plasma as a new technology in various fields of food and agriculture can provide effective and suitable solutions in order to promote the goals of the food industry, especially in the areas of mycotoxin elimination.

It can be deduced from Lin/Log charts and tables that aflatoxin B1 decontamination was performed with the help of compression of argon gas over time and the mean initial concentration of samples was significantly comparable with the mean concentration after each plasma radiation. It was concluded that the aflatoxin B1 detoxification was successful in all samples, indicating the high efficiency of the cold atmospheric plasma jet, especially in the food industry.

\section{REFERENCES}

1. C. Paulussen, J. E. Hallsworth, S. Álvarez-Pérez, W. C. Nierman, P. G. Hamill, D. Blain, H. Rediers, B. Lievens, Microbial Biotechnology, 10, 296 (2017).

2. D. W. Denning, Clin. Infect. Dis., 26, 781 (1998).

3. B. H. Segal, N. Engl. J. Med., 360, 1870 (2009).

4. R. M. Duran, J.W. Cary, A. M. Calvo, Appl. Microbiol. Biotechnol., 73, 1158 (2007).

5. A. M. Abdelhamid, Arch. Tierernahr., 40, 647 (1990).

6. J. W. Bennett, M. Klich, Clin. Microbiol. Rev., 16, 497 (2003).

7. N. Chamkouri, S., Khodadoust, F. Ghalavandi, Journal of Chromatographic Science, 53, 10 (2015).

8. C. P. Wild, Y. Y. Gong, Carcinogenesis, 31, 71, (2010).

9. M. F. Dutton, K. Ehrlich, J. W. Bennett, Appl. Environ. Microbiol., 49, 1392 (1985).

10. L. A. Loeb, C. C. Harris, Cancer Research, 68, 6863 (2008).

11. M. C. Smith, S. Madec, E. Coton, N. Hymery, Toxins, 8, 94 (2016).

12. K. Matra, S. Wongkuan, Procedia Computer Science, 86, 313 (2016).

13. A. Akbari, N. Chamkouri, A. Zadabdollah, Oriental Journal of Chemistry, 32, 6 (2016).

14. J. Napp, G. Daeschlein, M. Napp, S. von Podewils, D. Gümbel, R. Spitzmueller, P. Fornaciari, P. Hinz, M. Jünger, GMS Hygiene and Infection Control, 10, Doc08 (2015).

15. M. G. Kong, G. Kroesen, G. Morfill, T. Nosenko, T. Shimizu, J. van Dijk, J. L. Zimmermann, New Journal of Physics, 11, 115012 (2009).

16. M. Moisan, J. Barbeau, S. Moreau, J. Pelletier, M. Tabrizian, L. H. Yahia, Int. J. Pharm., 226, 1 (2001).

17. M. Laroussi, Plasma Processes and Polymers, 2, 391 (2005).

18. P. Basaran, N. Basaran-Akgul, L. Oksuz, Food Microbiol., 25, 626 (2008).

19. Y. Devi, Food Control, 77, 187 (2017).

20. S. A. Ouf, A. H. Basher, A. A. H. Mohamed, J. Sci. Food Agric., 95, 3204 (2015). 